\title{
CMTM6 significantly relates to PD-L1 and predicts the prognosis of gastric cancer patients
}

\author{
Xin Li ${ }^{\text {Equal first author, } 1}$, Ling Chen ${ }^{\text {Equal first author, } 2}{ }^{,}$Chuan $\mathbf{G u}^{3}$, Qiaoli Sun ${ }^{\text {Corresp., } 4}$, Jia Li ${ }^{\text {Corresp. } 5}$ \\ 1 Department of Oncology, Longhua Hospital, Shanghai University of Traditional Chinese Medicine(TCM), shanghai, China \\ Department of Oncology, Yueyang Hospital of Integrated Traditional Chinese and Western Medicine, Shanghai University of Traditional Chinese \\ Medicine(TCM), shanghai, China \\ 3 Department of plastic and reconstructive surgery, Shanghai Ninth People's Hospital, School of Medicine, Shanghai Jiaotong University, shanghai, China \\ 4 Institute of Digestive Diseases, Longhua Hospital, Shanghai University of Traditional Chinese Medicine(TCM), shanghai, China \\ 5 Department of Integrated Chinese and Western Medicine, Affiliated Cancer Hospital of Zhengzhou University and Henan Cancer Hospital, Zhengzhou, \\ China \\ Corresponding Authors: Qiaoli Sun, Jia Li \\ Email address: sunny5sunny@163.com, lijiacancerdoctor@126.com
}

BACKGROUND: The CKLF-like MARVEL transmembrane domain containing 6(CMTM6) is a key regulator of the programmed death receptor ligand-1(PD-L1) protein. However, the usefulness of CMTM6 expression as a prognostic indicator and the relationship between CMTM6 and PD-L1 expression in gastric cancer $(\mathrm{GC})$ remains unclear.

OBJECTIVES: We evaluated the expression and prognostic implications of CMTM6 in GC tissue and its relationship with PD-L1 expression.

PATIENTS AND METHODS: The protein expressions of CMTM6 and PD-L1 were detected in 122 cases of postoperative GC tissue using immunohistochemical (IHC) assays. Kaplan-Meier survival analysis was used to calculate the survival probability and a log-rank test was used to compare the survival curves. Univariate and multivariate Cox proportional hazard regression analyses were used to evaluate the clinically-related factors associated with survival. Pearson's correlation was used to determine the correlation analysis and estimate the statistical significance. The univariate and multivariate logistic regression analyses were used to analyze the relationship between clinically-related factors and PD-L1 expression.

RESULTS: Kaplan-Meier survival analysis showed that patients with high CMTM6 expression had shorter overall survival (OS) than those with low expression $(P<0.001)$. The expression of CMTM6 was an independent risk factor for prognosis in multivariate Cox proportional hazard regression analyses (HR:2.221, Cl\% 1.36-3.628, $P=0.001$ ). The OS of patients with positively expressed PD-L1 was significantly shorter than those with negatively expressed PD-L1 $(P=0.003)$. The expression of CMTM6 was significantly related to the positive expression of PD-L1 in gastric cancer tissues $(r=0.186, P=0.041)$. The expression of CMTM6 was the independent risk factor for PD-L1 expression in multivariate logistic regression analysis (OR:2.538, $\mathrm{Cl} \%$ 1.128-5.714, $P=0.024$ ).

CONCLUSION: CMTM6 expression is significantly related to PD-L1 and may be a useful prognostic indicator and a specific therapeutic target for cancer immunotherapy for GC patients. 
1 Title: CMTM6 significantly relates to PD-L1 and predicts the prognosis of gastric cancer patients

2 Author order: $\mathrm{Xin} \mathrm{Li}^{1 \#}$, Ling Chen²\#, Chuan $\mathrm{Gu}^{3}$, Qiaoli $\mathrm{Sun}^{4 *}, \mathrm{Jia} \mathrm{Li}^{5 *}$

\section{Author affiliations:}

$4{ }^{1}$ Department of Oncology, Longhua Hospital, Shanghai University of Traditional Chinese Medicine (TCM),

5 Shanghai 200032, China.

$6 \quad{ }^{2}$ Department of Oncology, Yueyang Hospital of Integrated Traditional Chinese and Western Medicine,

7 Shanghai University of Traditional Chinese Medicine (TCM), Shanghai 200437, China.

$8{ }^{3}$ Department of plastic and reconstructive surgery, Shanghai Ninth People's Hospital, School of

9 Medicine, Shanghai Jiaotong University, Shanghai 200011, China.

$10{ }^{4}$ Institute of Digestive Diseases, Longhua Hospital, Shanghai University of Traditional Chinese Medicine

11 (TCM), Shanghai 200032, China.

$12{ }^{5}$ Department of Integrated Chinese and Western Medicine, Affiliated Cancer Hospital of Zhengzhou

13 University and Henan Cancer Hospital, Zhengzhou, Henan 450008, China.

14 \#These authors contributed equally to this work.

15 *Corresponding authors:

16 Jia Li

17 Department of Integrated Chinese and Western Medicine, Affiliated Cancer Hospital of Zhengzhou University

18 and Henan Cancer Hospital, Zhengzhou, Henan 450008, China.

19 E-mail: lijiacancerdoctor@126.com

20 Qiaoli Sun

Peer] reviewing PDF | (2020:03:46767:1:1:NEW 17 Jun 2020) 
21 Institute of Digestive Diseases, Longhua Hospital, Shanghai University of Traditional Chinese Medicine

22 (TCM), Shanghai 200032, China.

23 E-mail: sunny5sunny@,163.com

24 ABSTRACT:

25

BACKGROUND: The CKLF-like MARVEL transmembrane domain containing 6(CMTM6) is a key regulator of the programmed death receptor ligand-1(PD-L1) protein. However, the usefulness of CMTM6 expression as a prognostic indicator and the relationship between CMTM6 and PD-L1 expression in gastric cancer (GC) remains unclear.

OBJECTIVES: We evaluated the expression and prognostic implications of CMTM6 in GC tissue and its relationship with PD-L1 expression.

PATIENTS AND METHODS: The protein expressions of CMTM6 and PD-L1 were detected in 122 cases of postoperative GC tissue using immunohistochemical (IHC) assays. Kaplan-Meier survival analysis was used to calculate the survival probability and a log-rank test was used to compare the survival curves. Univariate and multivariate Cox proportional hazard regression analyses were used to evaluate the clinically-related factors associated with survival. Pearson's correlation was used to determine the correlation analysis and estimate the statistical significance. The univariate and multivariate logistic regression analyses were used to analyze the relationship between clinically-related factors and PD-L1 expression.

RESULTS: Kaplan-Meier survival analysis showed that patients with high CMTM6 expression had shorter overall survival (OS) than those with low expression $(P<0.001)$. The expression of CMTM6 was an independent risk factor for prognosis in multivariate Cox proportional hazard regression analyses (HR:2.221, $\mathrm{CI} \% 1.36-3.628, P=0.001)$. The OS of patients with positively expressed PD-L1 was significantly shorter than 
42 those with negatively expressed PD-L1 $(P=0.003)$. The expression of CMTM6 was significantly related to the

43 positive expression of PD-L1 in gastric cancer tissues $(r=0.186, P=0.041)$. The expression of CMTM6 was the

44 independent risk factor for PD-L1 expression in multivariate logistic regression analysis (OR:2.538, CI\%

$451.128-5.714, P=0.024)$.

46 CONCLUSION: CMTM6 expression is significantly related to PD-L1 and may be a useful prognostic

47 indicator and a specific therapeutic target for cancer immunotherapy for GC patients.

48

\section{Introduction}

Gastric cancer is one of the most common malignant cancers of the digestive tract in the world [1]. The majority of GC patients in China are diagnosed in the advanced stages of the disease [2-3]. The 5-year survival rate for patients with advanced gastric cancer who receive comprehensive surgery-based treatment is less than $30 \%$ [4]. Gastric cancer is commonly treated using chemotherapy and anti-HER-2 targeted therapy, which have limited efficacies [5]. Immune checkpoint inhibitors (ICIs) are relatively new in the treatment and diagnosis of many cancers but they have demonstrated unprecedented clinical efficacy in multiple types of cancer and have good clinical application prospects in gastric cancer [6-9]. However, immunotherapy is only effective in a small proportion of gastric cancers and the expression of PD-L1 on tumor cells or tumorinfiltrating immune cells does not accurately predict for the patient's response to PD-1/PD-L1 inhibitors [1013]. Therefore, new immune-related therapeutic targets must be identified and their relationship with PD-L1 expression must be explored.

Two recent papers have identified CMTM6, which is a PD-L1 regulatory factor that plays an important role in inhibiting T-cell activation and anti-tumor responses. CMTM6 displays specificity for PD-L1 and promotes the expression of PD-L1 in tumor cells by T-cells. The depletion of CMTM6 decreases PD-L1 without 
63

64

65

66

67

68

69

70

71

72

compromising the cell surface expression of MHC class I [14-15]. The role of CMTM6 in anti-tumor

immunity and stabilization of the expression of PD-L1 has prompted us to explore its role in gastric cancer.

In this study, we evaluated the expression of CMTM6 and PD-L1 in 122 GC samples to investigate the

prognostic success of CMTM6 and its relation to PD-L1 for GC patients.

\section{Materials and Methods}

\section{Tissue samples}

We collected 122 tissue samples from gastric cancer patients who were surgically treated at the First Affiliated Hospital of Naval Military Medical University from December 2000 to June 2002. The study's follow-up deadline was October 31, 2007. Patients did not receive any radiation or chemotherapy before surgery. Tissue samples and clinical information were collected after patient consent was obtained and our study was approved by the ethics committee of the First Affiliated Hospital of Naval Military Medical University (ID: CHEC2014098).

\section{Immunohistochemistry staining and results interpretation}

All 122 gastric cancer samples were processed into tissue chips. The CMTM6 antibody was obtained from Novus Biologicals (NBP1-31183, United States) [16]. The PD-L1 antibody for IHC was purchased from Arigo Biolaboratories (ARG57681, Taiwan, China) [17]. The IHC kit was obtained from Maixin Biotechnologies (KIT-9730, Fuzhou, China) and was used according the manufacturer's instructions. The tissue chip was heated on a 63-degree baking sheet for 1 hour. It was then dewaxed and hydrated in a fully automatic dyeing machine. The antigens were retrieved from the sample using a high-pressure heat repair method and the primary antibody was added (PD-L1 antibody 1: 50 and CMTM6 antibody 1: 200). The sample was returned to heat for $1 \mathrm{~h}$ at $37^{\circ} \mathrm{C}$ and a goat anti-rabbit secondary antibody was added and heated for $30 \mathrm{~min}$ at $37^{\circ} \mathrm{C}$. 
84

85

86

87

88

89

90

91

92

93

94

95

96

97

98

99

100

101

102

103

104

3,3-N-Diaminobenzidine Tertrahydrochloride (DAB) was developed for 1 minute, hematoxylin was

counterstained for 10 minutes, and the film was sealed.

Five fields from each specimen were randomly selected for analysis. The staining results were scored

according to the Rahmen criteria and all of the IHC results were reviewed by two senior pathologists. Scores

were used to assess CMTM6 expression according to the positive rate of staining: 0 points (negative), 1 point

(1-25\%), 2 points $(26 \%-50 \%), 3$ points $(51-75 \%)$, or 4 points $(76 \%-100 \%)$. X-tile plots were created by

dividing CMTM6 expression into two populations: low- and high-level expression. All possible divisions of

CMTM6 expression were assessed. Associations could be calculated at each division by log-rank test for

survival. The data were represented graphically in a right-triangular grid where each point (pixel) represented

the data from a given set of divisions. Data along the hypotenuse represented results from a single cut-point

that divided the data into high or low subsets [18]. 0.5 was the best cutoff value of CMTM6 expression for this

set of samples. PD-L1 expression was considered to be positively expressed when the proportion of cells with

membranous staining was $>1 \%$ in neoplastic cells [19].

\section{Statistical analysis}

The Kaplan-Meier method was used to calculate survival probability and the log-rank test was used to compare

survival curves. The univariate and multivariate Cox proportional hazard regression analyses were used to

evaluate the clinically-related factors associated with survival. The correlation analysis and estimated statistical

significance was determined according to Pearson's correlation. The relationship between the clinically-related

factors and PD-L1 expression were analyzed using univariate and multivariate logistic regression analyses. All

statistical tests were two-sided, and $P<0.05$ was considered to be statistically significant. SPSS 20.0 (SPSS,

IL, Chicago) was used for all statistical data analysis.

Peer) reviewing PDF | (2020:03:46767:1:1:NEW 17 Jun 2020) 
105

106

107

108

109

110

111

112

113

114

115

116

117

118

119

120

121

122

123

124

125

\section{Results}

\section{Clinicopathological characteristics of GC patients}

The clinical characteristics of 122 gastric cancer patients are summarized in Table $1.52(42.6 \%)$ patients were diagnosed at less than 60 years of age; $89(73.0 \%)$ patients were male. $26(21.3 \%)$ patients had tumors $\leq 3 \mathrm{~cm}$, $68(55.7 \%)$ patients had tumors $3-6 \mathrm{~cm}$, and $28(23.0 \%)$ patients had tumors $>6 \mathrm{~cm} .42$ patients $(34.4 \%)$ had TNM stage I tumors, 48 patients (39.4\%) had TNM stage II tumors, and 32 patients $(26.2 \%)$ had TNM stage

III tumors. 80 patients $(65.6 \%)$ had tumors with pathological grades I-II and 42 patients $(34.4 \%)$ had grade III tumors according to the AJCC criteria staging system [3]. The pathological morphology of patients with adenocarcinoma or a different tumor type was $107(87.7 \%)$ and $15(12.3 \%)$ respectively.

\section{Expression of CMTM6 and PD-L1 in GC tissues}

We detected the expression of CMTM6 and PD-L1 in 122 gastric cancer tissues using an IHC assay. As shown in Figure 1, CMTM6 is located mainly in the cell membrane and cytoplasm and the expression of PD-L1 was observed on the cell membrane. The low- and high-expressions of CMTM6 in gastric cancer tissues were $46.7 \%(57 / 122)$ and $53.3 \%$ (65/122), respectively, and the negative and positive expressions of PD-L1 were 35 (28.7\%) and $87(71.3 \%)$, respectively (Table 1$)$.

\section{The prognostic value of CMTM6 expression in patients with GC}

The cut-off level for CMTM6 expression was determined using X-tile software (Figure 2A). Kaplan-Meier survival analysis showed that patients with high CMTM6 expression had shorter overall survival (OS) than these with low expression (Figure $2 \mathrm{~B}, P<0.001$ ). Univariate and multivariate Cox proportional hazard regression analyses were used to evaluate the clinically-related factors associated with survival. CMTM6 expression was an independent risk factor for prognosis (Table 2, HR:2.221, CI\% 1.36-3.628, $P=0.001$ ). 
126

127

128

129

130

131

132

133

134

135

136

137

138

139

140

141

142

143

144

145

146

\section{Relationship between CMTM6 and PD-L1 in GC}

Patients with positively expressed PD-L1 has a significantly shorter OS than those with negatively expressed

PD-L1 (Figure 3A) $(P=0.003)$. CMTM6 expression was significantly related to PD-L1 positivity in gastric cancer tissues (Figure 3B) $(\mathrm{r}=0.186, P=0.041)$. Age, sex, tumor size, TNM stage, grade, histology, and CMTM6 expression were selected in the univariate and multivariate logistic regression analysis to further evaluate the relationship between the positive expression of PD-L1 and other clinically-related factors. The results showed that only CMTM6 expression was a risk factor of PD-L1 expression (Table 3) (OR:2.538, CI\% $1.128-5.714, P=0.024)$.

\section{Discussion}

CMTM6 belongs to a family of proteins made up of eight members (CMTM1-4 on the 16q21-22 chromosomal region, CMTM5 on the 14q11 region, and CMTM6-8 on the 3p22 region) that play a role in epigenetic regulation, embryonic development, and tumorigenesis [20-22]. CMTM6 is not required for PD-L1 maturation but co-localizes with PD-L1 at the plasma membrane and in recycling endosomes where it prevents PD-L1 from being targeted for lysosome-mediated degradation [14]. CMTM6 was identified as a major regulator of PD-L1, a key immunological checkpoint, and a potential therapeutic target for tumor cell immune evasion.

There have been reports on CMTM6 for its regulation of PD-L1 and its function at the genetic and cellular levels for different cell lines. However, the usefulness of CMTM6 in clinical cancer samples has yet to be determined. We reported the prognostic value of CMTM6 and its relationship to PD-L1 in GC for the first time in our study. High CMTM6 expression was associated with shorter overall survival (OS) versus low expression $(P<0.001)$. Multivariate analysis confirmed the prognostic value (HR:2.221, CI\% 1.36-3.628, $P=0.001$ ). The expression of CMTM6 was significantly related to the positive presence of PD-L1 in gastric 
147

cancer tissues ( $\mathrm{r}=0.186, P=0.041)$. The expression of CMTM6 was the only independent risk factor of PD-L1 expression found by multivariate logistic regression analysis (OR:2.538, CI\% 1.128-5.714, $P=0.024)$.

There are few studies reporting on CMTM6 expression and prognosis in other cancers. In primary pancreatic ductal adenocarcinoma (PDAC), a high CMTM6 expression was associated with shorter overall survival (OS).

In the case of low expression, expression of CMTM6 also increased the prognostic value of PD-L1 expression [23]. CMTM6 expression was associated with poor prognosis in hepatocellular carcinoma (HCC). The expression of CMTM6 was downregulated and was correlated with the AFP level and tumor metastasis of HCC patients [16]. CMTM6 overexpression was associated with poor prognosis in gliomas. CMTM6 is important for regulating T-cell activation and antitumor responses and is a promising target for developing immunotherapy of gliomas [24]. A high CMTM6 expression was associated with reduced survival time and may be a strong indicator of poor prognosis in different cancer types.

CMTM6 maintains the stability of the PD-L1 cell surface expression and plays a key role in the survival of PD-L1 [15]. CMTM6 expression was positively correlated with PD-L1 in immunohistochemical and mRNA expression data of non-small cell lung cancer [25-26]. Preventing CMTM6 from binding with PD-L1 may recover the existing immunosuppression response and serve as a promising target for immunotherapy. Zugazagoitia et al found that high CMTM6 and PD-L1 co-expression in stromal cells, but not tumor cells, of lung cancer was significantly associated with longer overall survival in treated patients. However, this effect was not observed in the absence of immunotherapy [27]. Koh et al. also found that CMTM6 expression may be a promising tool for therapeutic decision-making regarding PD-1 inhibitors [25]. Zhao et al. discovered a statistically significant interaction between CMTM6 and CD274 (PD-L1) indicating that CMTM4/6 may be a new therapeutic target for type I renal clear cell carcinoma (RCC) patients who are resistant to immune 
168

169

170

171

172

173

174

175

176

177

178

179

180

181

182

183

184

185

186

187

188

189

190

191

checkpoint blockade (ICB) [28]. CMTM6 expression was positively correlated with PD-L1 in GC, making it a

promising immunotherapy target for gastric cancer.

This study also has certain limitations. Firstly, we used IHC to detect CMTM6 and PD-L1 expressions.

However, this process is limited by positive criteria, the definition of cut-off values, and the specificity and

reproducibility of the antibodies used. Secondly, due to some patients lacked the pathological data of Lauren

classification, we did not perform a correlation analysis between CMTM6 and Laurent classification. Thirdly,

because of the influence of various clinical factors, such as pathological type, tumor size, etc., TNM stage is

not a risk factor affecting the prognosis of gastric cancer patients.

Conclusion: CMTM6 expression is significantly related to PD-L1 and may be a useful prognostic indicator

and a specific therapeutic target for cancer immunotherapy for GC patients.

Acknowledgments: Not applicable.

\section{References}

[1] Globocan 2018.International Agency for Research Cancer. Available at http://gco.iarc.fr/today/home.

[2] Qiu, M.Z., Wang, Z.X., Zhou, Y.X., Yang, D.J., Wang, F.H., and Xu, R.H. 2018. Proposal for a New TNM

Stage based on the 7(th) and 8(th) American Joint Committee on Cancer pTNM Staging Classification for

Gastric Cancer. Journal of Cancer 9:3570-3576. DOI:10.7150/jca.26351

[3] Liu, J.Y., Peng, C.W., Yang, X.J., Huang, C.Q., and Li, Y. 2018. The prognosis role of AJCC/UICC 8(th)

edition staging system in gastric cancer, a retrospective analysis. American Journal of Translational Research 10:292-303. PMID: 29423014

[4] Katai, H., Ishikawa, T., Akazawa, K., Isobe, Y., Miyashiro, I., Oda, I., Tsujitani, S., Ono, H., Tanabe, S., Fukagawa, T., Nunobe, S., Kakeji, Y., and Nashimoto, A. 2018. Five-year survival analysis of surgically resected gastric cancer cases in Japan: a retrospective analysis of more than 100,000 patients from the nationwide registry of the Japanese Gastric Cancer Association (2001-2007). Gastric Cancer 21:144-154. DOI:10.1007/s10120017-0716-7

Peer] reviewing PDF | (2020:03:46767:1:1:NEW 17 Jun 2020) 
192 [5] Wagner, A.D., Unverzagt, S., Grothe, W., Kleber, G., Grothey, A., Haerting, J., and Fleig, W.E. 2010.

193 Chemotherapy for advanced gastric cancer. Cochrane Database Syst Rev:D4064.

194 DOI:10.1002/14651858.CD004064.pub3

195 [6] Sharma, P., and Allison, J.P. 2015. The future of immune checkpoint therapy. SCIENCE 348:56-61.

196 DOI:10.1126/science.aaa8172

197 [7] Fuchs, C.S., Doi, T., Jang, R.W., Muro, K., Satoh, T., Machado, M., Sun, W., Jalal, S.I., Shah, M.A., 198 Metges, J.P., Garrido, M., Golan, T., Mandala, M., Wainberg, Z.A., Catenacci, D.V., Ohtsu, A., Shitara, K., 199 Geva, R., Bleeker, J., Ko, A.H., Ku, G., Philip, P., Enzinger, P.C., Bang, Y.J., Levitan, D., Wang, J., Rosales, 200 M., Dalal, R.P., and Yoon, H.H. 2018. Safety and Efficacy of Pembrolizumab Monotherapy in Patients With 201 Previously Treated Advanced Gastric and Gastroesophageal Junction Cancer: Phase 2 Clinical KEYNOTE202 059 Trial. JAMA Oncology 4:e180013. DOI:10.1001/jamaoncol.2018.0013

203 [8] Shitara, K., Ozguroglu, M., Bang, Y.J., Di Bartolomeo, M., Mandala, M., Ryu, M.H., Fornaro, L., 204 Olesinski, T., Caglevic, C., Chung, H.C., Muro, K., Goekkurt, E., Mansoor, W., McDermott, R.S., Shacham205 Shmueli, E., Chen, X., Mayo, C., Kang, S.P., Ohtsu, A., and Fuchs, C.S. 2018. Pembrolizumab versus 206 paclitaxel for previously treated, advanced gastric or gastro-oesophageal junction cancer (KEYNOTE-061): a 207 randomised, open-label, controlled, phase 3 trial. LANCET 392:123-133. DOI:10.1016/S0140-

$208 \quad 6736(18) 31257-1$

209 [9] Kang, Y.K., Boku, N., Satoh, T., Ryu, M.H., Chao, Y., Kato, K., Chung, H.C., Chen, J.S., Muro, K., Kang, 210 W.K., Yeh, K.H., Yoshikawa, T., Oh, S.C., Bai, L.Y., Tamura, T., Lee, K.W., Hamamoto, Y., Kim, J.G., Chin, 211 K., Oh, D.Y., Minashi, K., Cho, J.Y., Tsuda, M., and Chen, L.T. 2017. Nivolumab in patients with advanced 212 gastric or gastro-oesophageal junction cancer refractory to, or intolerant of, at least two previous chemotherapy 213 regimens (ONO-4538-12, ATTRACTION-2): a randomised, double-blind, placebo-controlled, phase 3 trial.

214 LANCET 390:2461-2471. DOI:10.1016/S0140-6736(17)31827-5

215 [10] Braun, D.A., Burke, K.P., and Van Allen, E.M. 2016. Genomic Approaches to Understanding Response 216 and Resistance to Immunotherapy. CLINICAL CANCER RESEARCH 22:5642-5650. DOI:10.1158/1078217 0432.CCR-16-0066

218 [11] Guan, J., Lim, K.S., Mekhail, T., and Chang, C.C. 2017. Programmed Death Ligand-1 (PD-L1)

219 Expression in the Programmed Death Receptor-1 (PD-1)/PD-L1 Blockade: A Key Player Against Various 220

Cancers. ARCHIVES OF PATHOLOGY \& LABORATORY MEDICINE 141:851-861.

Peer] reviewing PDF | (2020:03:46767:1:1:NEW 17 Jun 2020) 
221

222

223

224

225

226

227

228

229

230

231

232

233

234

235

236

237

238

239

240

241

242

243

244

245

246

247

248

249

DOI:10.5858/arpa.2016-0361-RA

[12] Wang, Q., and Wu, X. 2017. Primary and acquired resistance to PD-1/PD-L1 blockade in cancer treatment. INTERNATIONAL IMMUNOPHARMACOLOGY 46:210-219.

DOI:10.1016/j.intimp.2017.03.015

[13] Pardoll, D.M. 2012. The blockade of immune checkpoints in cancer immunotherapy. NATURE

REVIEWS CANCER 12:252-264. DOI:10.1038/nrc3239

[14] Burr, M.L., Sparbier, C.E., Chan, Y.C., Williamson, J.C., Woods, K., Beavis, P.A., Lam, E., Henderson, M.A., Bell, C.C., Stolzenburg, S., Gilan, O., Bloor, S., Noori, T., Morgens, D.W., Bassik, M.C., Neeson, P.J., Behren, A., Darcy, P.K., Dawson, S.J., Voskoboinik, I., Trapani, J.A., Cebon, J., Lehner, P.J., and Dawson, M.A. 2017. CMTM6 maintains the expression of PD-L1 and regulates anti-tumour immunity. NATURE 549:101-105. DOI:10.1038/nature23643

[15] Mezzadra, R., Sun, C., Jae, L.T., Gomez-Eerland, R., de Vries, E., Wu, W., Logtenberg, M., Slagter, M., Rozeman, E.A., Hofland, I., Broeks, A., Horlings, H.M., Wessels, L., Blank, C.U., Xiao, Y., Heck, A., Borst, J., Brummelkamp, T.R., and Schumacher, T. 2017. Identification of CMTM6 and CMTM4 as PD-L1 protein regulators. NATURE 549:106-110. DOI:10.1038/nature23669

[16] Zhu, X., Qi, G., Li, C., Bei, C., Tan, C., Zhang, Y., Shi, W., Zeng, W., Kong, J., Fu, Y., and Tan, S. 2019. Expression and Clinical Significance of CMTM6 in Hepatocellular Carcinoma. DNA AND CELL BIOLOGY 38:193-197. DOI:10.1089/dna.2018.4513

[17] Freeman, G.J., Long, A.J., Iwai, Y., Bourque, K., Chernova, T., Nishimura, H., Fitz, L.J., Malenkovich, N., Okazaki, T., Byrne, M.C., Horton, H.F., Fouser, L., Carter, L., Ling, V., Bowman, M.R., Carreno, B.M., Collins, M., Wood, C.R., and Honjo, T. 2000. Engagement of the PD-1 immunoinhibitory receptor by a novel B7 family member leads to negative regulation of lymphocyte activation. JOURNAL OF EXPERIMENTAL MEDICINE 192:1027-1034. DOI:10.1084/jem.192.7.1027

[18] Camp, R.L., Dolled-Filhart, M., and Rimm, D.L. 2004. X-tile: a new bio-informatics tool for biomarker assessment and outcome-based cut-point optimization. CLINICAL CANCER RESEARCH 10:7252-7259. DOI:10.1158/1078-0432.CCR-04-0713

[19] Calderaro, J., Rousseau, B., Amaddeo, G., Mercey, M., Charpy, C., Costentin, C., Luciani, A., Zafrani, E.S., Laurent, A., Azoulay, D., Lafdil, F., and Pawlotsky, J.M. 2016. Programmed death ligand 1 expression in hepatocellular carcinoma: Relationship With clinical and pathological features. HEPATOLOGY 64:2038-

Peer] reviewing PDF | (2020:03:46767:1:1:NEW 17 Jun 2020) 
250

251

252

253

254

255

256

257

258

259

260

261

262

263

264

265

266

267

268

269

270

271

272

273

274

275

276

277

278

2046. DOI:10.1002/hep.28710

[20] Han, W., Ding, P., Xu, M., Wang, L., Rui, M., Shi, S., Liu, Y., Zheng, Y., Chen, Y., Yang, T., and Ma, D. 2003. Identification of eight genes encoding chemokine-like factor superfamily members 1-8 (CKLFSF1-8) by in silico cloning and experimental validation. GENOMICS 81:609-617. DOI:10.1016/s08887543(03)00095-8

[21] Sanchez-Pulido, L., Martin-Belmonte, F., Valencia, A., and Alonso, M.A. 2002. MARVEL: a conserved domain involved in membrane apposition events. TRENDS IN BIOCHEMICAL SCIENCES 27:599-601.

DOI:10.1016/s0968-0004(02)02229-6

[22] Yafune, A., Kawai, M., Itahashi, M., Kimura, M., Nakane, F., Mitsumori, K., and Shibutani, M. 2013.

Global DNA methylation screening of liver in piperonyl butoxide-treated mice in a two-stage

hepatocarcinogenesis model. TOXICOLOGY LETTERS 222:295-302. DOI:10.1016/j.toxlet.2013.08.006

[23] Mamessier, E., Birnbaum, D.J., Finetti, P., Birnbaum, D., and Bertucci, F. 2018. CMTM6 stabilizes PD-

L1 expression and refines its prognostic value in tumors. Ann Transl Med 6:54. DOI:10.21037/atm.2017.11.26

[24] Guan, X., Zhang, C., Zhao, J., Sun, G., Song, Q., and Jia, W. 2018. CMTM6 overexpression is associated with molecular and clinical characteristics of malignancy and predicts poor prognosis in gliomas.

EBioMedicine 35:233-243. DOI:10.1016/j.ebiom.2018.08.012

[25] Koh, Y.W., Han, J.H., Haam, S., Jung, J., and Lee, H.W. 2019. Increased CMTM6 can predict the clinical response to PD-1 inhibitors in non-small cell lung cancer patients. OncoImmunology 8:e1629261.

DOI:10.1080/2162402X.2019.1629261

[26] Gao, F., Chen, J., Wang, J., Li, P., Wu, S., Wang, J., and Ji, Y. 2019. CMTM6, the newly identified PDL1 regulator, correlates with PD-L1 expression in lung cancers. Biochem Biophys Rep 20:100690.

DOI:10.1016/j.bbrep.2019.100690

[27] Zugazagoitia, J., Liu, Y., Toki, M., McGuire, J., Ahmed, F.S., Henick, B.S., Gupta, R., Gettinger, S.N., Herbst, R.S., Schalper, K.A., and Rimm, D.L. 2019. Quantitative Assessment of CMTM6 in the Tumor Microenvironment and Association with Response to PD-1 Pathway Blockade in Advanced-Stage Non-Small Cell Lung Cancer. Journal of Thoracic Oncology 14:2084-2096. DOI:10.1016/j.jtho.2019.09.014

[28] Zhao, W., Zhao, F., Yang, K., Lu, Y., Zhang, Y., Wang, W., Xie, H., Deng, K., Yang, C., Rong, Z., Hou, Y., and Li, K. 2019. An immunophenotyping of renal clear cell carcinoma with characteristics and a potential therapeutic target for patients insensitive to immune checkpoint blockade. JOURNAL OF CELLULAR 


\section{Figure legends}

Figure 1.The expression of CMTM6 and PD-L1 in GC tissues. A-C. Negative, weak positive and strong positive expression of CMTM6 (7×) D-F. Negative, weak positive and strong positive expression of PD-L1 $(7 \times)$

Figure 2. The prognostic value of CMTM6 expression in patients with GC. A. X-tile plots Pattern diagram. The vertical axis represents all possible "high" populations, with the size of the high population increasing from top to bottom. The horizontal axis represents all possible "low" populations, with the size of the low population increasing from left to right. Coloration of the plot represents the strength of the association at each division, ranging from low (black) to high (gray, or white). 0.5 is the best cutoff value of CMTM6 expression 
304 for this set of samples by X-title software. B. Kapla-Meier analysis of overall survival (OS) with variable

305 CMTM6 expression in GC patients. The black curve represents CMTM6 high expression group, and the gray

306 curve represents CMTM6 low expression group.

307 Figure 3. Relationship between CMTM6 and PD-L1 in GC.A. Kapla-Meier analysis of overall survival (OS)

308 with variable PD-L1expression in GC patients. The black curve represents PD-L1 positive expression group,

309 and the gray curve represents PD-L1 negative expression group. B. The correlation of CMTM6 and PD-L1

310 expression in the GC tissues.

311 Table legends

312 Table 1: The clinicopathological characteristics of GC patients.

313 Table 2: The univariate and multivariate Cox proportional hazard regression analyses between the clinical

314 related factors and survival in GC patients.

315 Table 3: The univariate and multivariate logistic regression analysis between the clinical related risk factors

316 and PD-L1 expression in GC patients. 
Figure 1

The expression of CMTM6 and PD-L1 in GC tissues.

A-C. Negative, weak positive and strong positive expression of CMTM6 (7x) D-F. Negative, weak positive and strong positive expression of PD-L1 (7x).

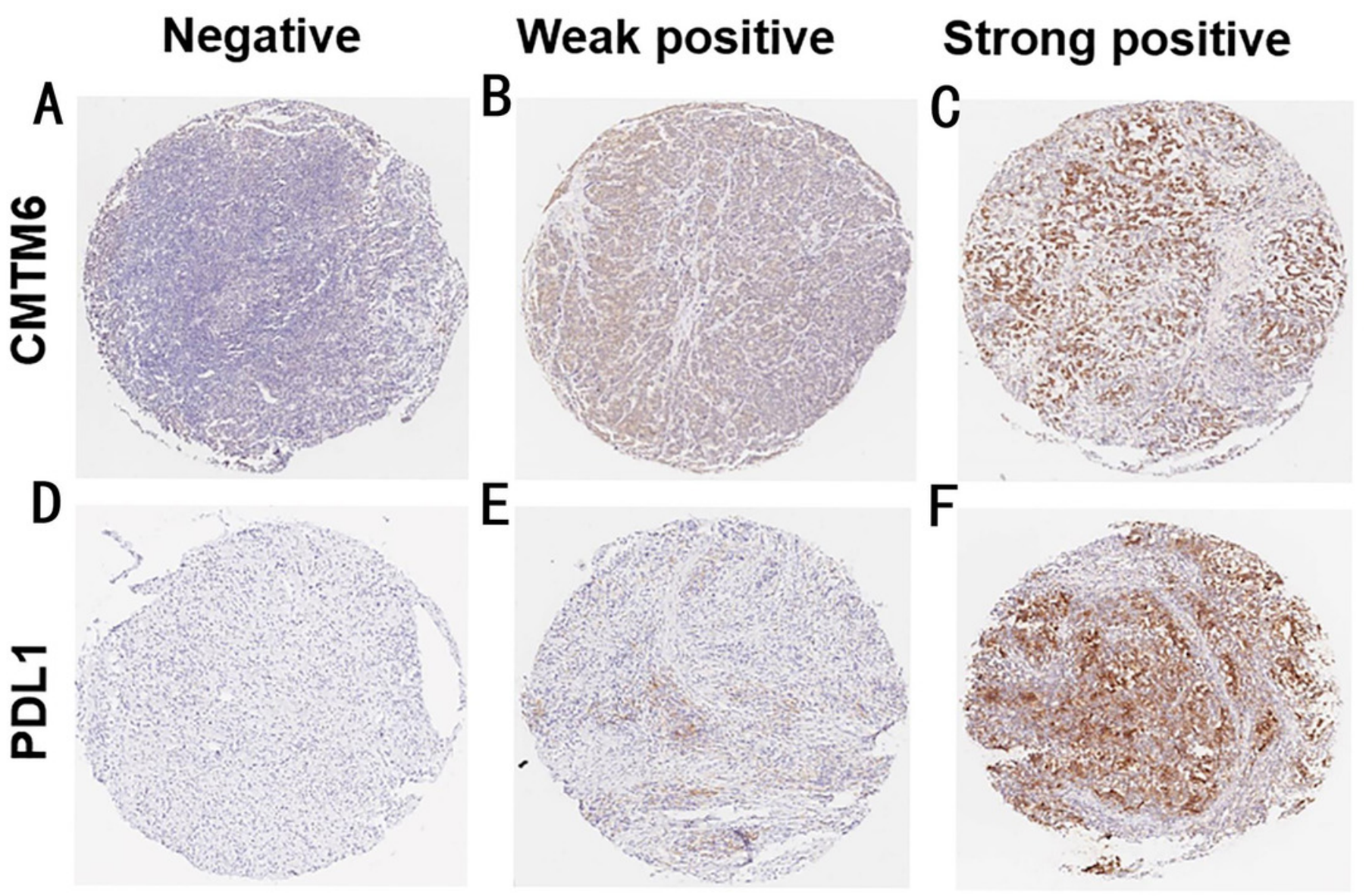




\section{Figure 2}

The prognostic value of CMTM6 expression in patients with GC.

A. X-tile plots Pattern diagram. The vertical axis represents all possible "high" populations, with the size of the high population increasing from top to bottom. The horizontal axis represents all possible "low" populations, with the size of the low population increasing from left to right. Coloration of the plot represents the strength of the association at each division, ranging from low ( black ) to high (gray, or white). 0.5 is the best cutoff value of CMTM6 expression for this set of samples by X-title software. B. Kapla-Meier analysis of overall survival (OS) with variable CMTM6 expression in GC patients. The black curve represents CMTM6 high expression group, and the gray curve represents CMTM6 low expression group.

A

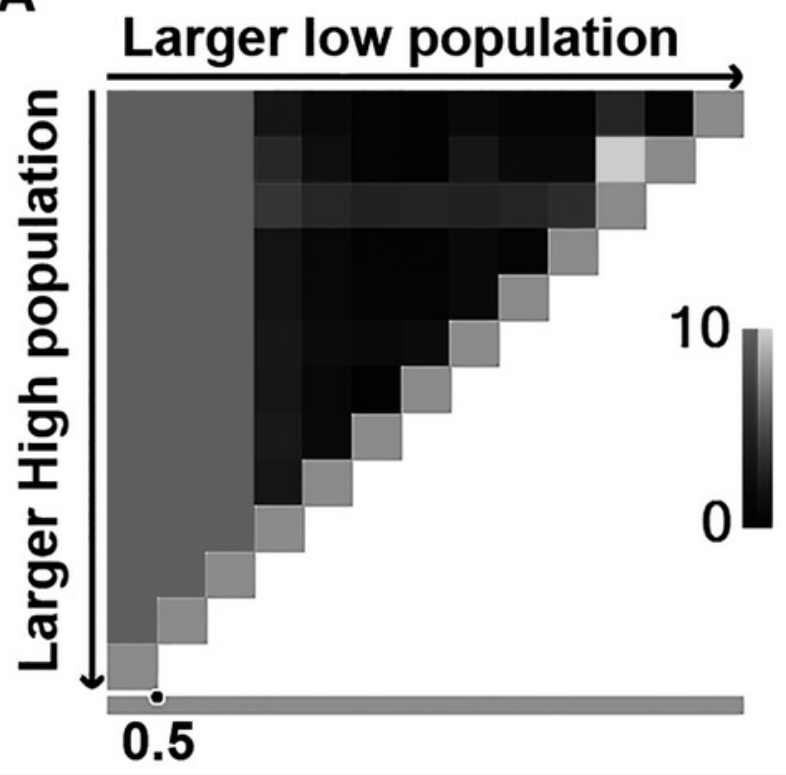

B

CMTM6

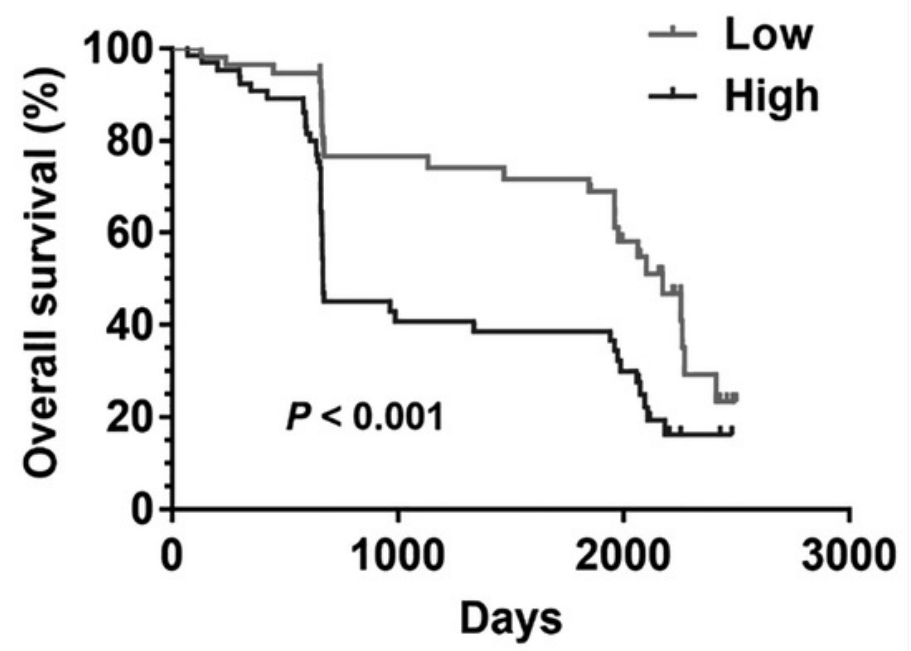


Figure 3

Relationship between CMTM6 and PD-L1 in GC.

A. Kapla-Meier analysis of overall survival (OS) with variable PD-Llexpression in GC patients. The black curve represents PD-L1 positive expression group, and the gray curve represents PD-L1 negative expression group. B. The correlation of CMTM6 and PD-L1 expression in the GC tissues.

A

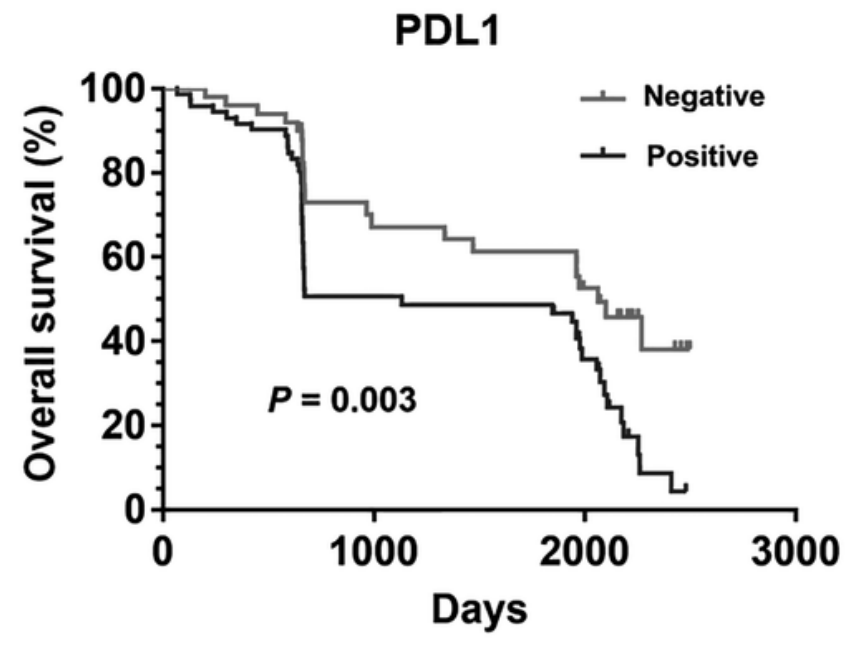

B

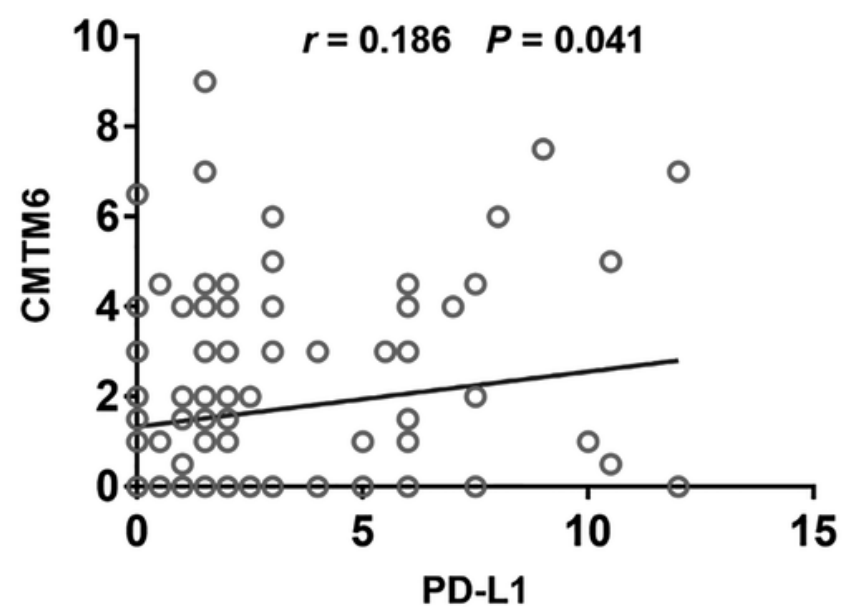




\section{Table $\mathbf{1}$ (on next page)}

Table 1: The clinicopathological characteristics of GC patients. 
1 Table 1

2

\begin{tabular}{|c|c|c|}
\hline Variables & Number & $\%$ \\
\hline \multicolumn{3}{|l|}{ Age(year) } \\
\hline$<60$ & 52 & 42.6 \\
\hline$\geq 60$ & 70 & 57.4 \\
\hline \multicolumn{3}{|l|}{ Sex } \\
\hline Male & 89 & 73.0 \\
\hline Female & 33 & 27.0 \\
\hline \multicolumn{3}{|l|}{ Tumor size(cm) } \\
\hline$\leq 3$ & 26 & 21.3 \\
\hline $3-6$ & 68 & 55.7 \\
\hline$\geq 6$ & 28 & 23.0 \\
\hline \multicolumn{3}{|l|}{ TNM stage (AJCC) } \\
\hline I & 42 & 34.4 \\
\hline II & 48 & 39.4 \\
\hline III & 32 & 26.2 \\
\hline \multicolumn{3}{|l|}{ Grade(AJCC) } \\
\hline $\mathrm{I}+\mathrm{II}$ & 80 & 65.6 \\
\hline III & 42 & 34.4 \\
\hline \multicolumn{3}{|l|}{ Histology } \\
\hline Adenocarcinoma & 107 & 87.7 \\
\hline Others & 15 & 12.3 \\
\hline \multicolumn{3}{|l|}{ СМТМ6 } \\
\hline Low & 57 & 46.7 \\
\hline High & 65 & 53.3 \\
\hline \multicolumn{3}{|l|}{ PDL1 } \\
\hline Negative & 35 & 28.7 \\
\hline Positive & 87 & 71.3 \\
\hline
\end{tabular}




\section{Table 2 (on next page)}

Table 2: The univariate and multivariate Cox proportional hazard regression analyses between the clinical related factors and survival in GC patients. 
1

2 Table 2

3

\begin{tabular}{|c|c|c|c|}
\hline \multirow[t]{2}{*}{ Variables } & \multirow{2}{*}{$\begin{array}{l}\text { Univariate analysis } \\
\text { Wald } \chi 2\end{array}$} & \multicolumn{2}{|c|}{ Multivariate analysis } \\
\hline & & HR (95\%CI) & $\boldsymbol{P}$ \\
\hline Age(year) & 0.185 & & 0.667 \\
\hline$<60$ & & Reference & \\
\hline$\geq 60$ & & $1.119(0.672-1.862)$ & 0.667 \\
\hline Sex & 0.206 & & 0.650 \\
\hline Male & & Reference & \\
\hline Female & & $0.881(0.51-1.522)$ & 0.650 \\
\hline Tumor size(cm) & 4.82 & & 0.090 \\
\hline$\leq 3$ & & Reference & \\
\hline $3-6$ & & $1.805(0.954-3.414)$ & 0.069 \\
\hline$\geq 6$ & & $1.088(0.493-2.402)$ & 0.834 \\
\hline TNM stage (AJCC) & 0.514 & & 0.773 \\
\hline I & & Reference & \\
\hline II & & $0.887(0.474-1.662)$ & 0.709 \\
\hline III & & $1.136(0.608-2.121)$ & 0.689 \\
\hline Grade (AJCC) & 0.436 & & 0.509 \\
\hline $\mathrm{I}+\mathrm{II}$ & & Reference & \\
\hline III & & $1.188(0.712-1.984)$ & 0.509 \\
\hline Histology & 2.599 & & 0.107 \\
\hline Adenocarcinoma & & Reference & \\
\hline Others & & $1.869(0.874-3.997)$ & 0.107 \\
\hline СМТМ6 & 10.157 & & 0.001 \\
\hline Low & & Reference & \\
\hline High & & $2.221(1.36-3.628)$ & 0.001 \\
\hline PDL1 & 1.256 & & 0.262 \\
\hline Negative & & Reference & \\
\hline Positive & & $1.409(0.774-2.566)$ & 0.262 \\
\hline
\end{tabular}




\section{Table 3 (on next page)}

Table 3: The univariate and multivariate logistic regression analysis between the clinical related risk factors and PD-L1 expression inGC patients. 
1

2 Table 3

3

\begin{tabular}{|c|c|c|c|}
\hline \multirow[t]{2}{*}{ Variables } & \multirow{2}{*}{$\begin{array}{l}\text { Univariate analysis } \\
\text { Wald } \chi 2\end{array}$} & \multicolumn{2}{|c|}{ Multivariate analysis } \\
\hline & & OR $(95 \% C I)$ & $\boldsymbol{P}$ \\
\hline Age(year) & 1.348 & & 0.246 \\
\hline$<60$ & & Reference & \\
\hline$\geq 60$ & & $1.634(0.713-3.741)$ & 0.246 \\
\hline Sex & 0.572 & & 0.45 \\
\hline Male & & Reference & \\
\hline Female & & $1.473(0.54-4.017)$ & 0.45 \\
\hline Tumor size(cm) & 3.592 & & 0.166 \\
\hline$\leq 3$ & & Reference & \\
\hline $3-6$ & & $2.507(0.946-6.645)$ & 0.065 \\
\hline$\geq 6$ & & $2.262(0.695-7.358)$ & 0.175 \\
\hline TNM stage (AJCC) & 2.719 & & 0.257 \\
\hline I & & Reference & \\
\hline II & & $0.561(0.2-1.568)$ & 0.27 \\
\hline III & & $1.392(0.421-4.597)$ & 0.588 \\
\hline Grade(AJCC) & 0.241 & & 0.624 \\
\hline $\mathrm{I}+\mathrm{II}$ & & Reference & \\
\hline III & & $0.786(0.3-2.059)$ & 0.624 \\
\hline Histology & 0.044 & & 0.834 \\
\hline Adenocarcinoma & & Reference & \\
\hline Others & & $0.862(0.215-3.464)$ & 0.834 \\
\hline СМТМ6 & 5.063 & & 0.024 \\
\hline Low & & Reference & \\
\hline High & & $2.538(1.128-5.714)$ & 0.024 \\
\hline
\end{tabular}

